ARTICLE

DOI: $10.1038 / \mathrm{s} 41467-018-05744-\mathrm{x}$

\title{
Light-induced mechanical response in crosslinked liquid-crystalline polymers with photoswitchable glass transition temperatures
}

\author{
Youfeng Yue ${ }^{1}$, Yasuo Norikane (iD ${ }^{1}$, Reiko Azumi ${ }^{1}$ \& Emiko Koyama ${ }^{1}$
}

Energy conversion of light into mechanical work is of fundamental interest in applications. In particular, diligent molecular design on nanoscale, in order to achieve efficient photomechanical effects on macroscopic scale, has become one of the most interesting study topics. Here, by incorporating a "photomelting" azobenzene monomer crosslinked into liquid crystalline (LC) networks, we generate photoresponsive polymer films that exhibit reversible photoswitchable glass transition temperatures $\left(T_{\mathrm{g}}\right)$ at room temperature $\left(\sim 20^{\circ} \mathrm{C}\right)$ and photomechanical actuations under the stimulus of UV/visible light. The trans-to-cis isomerization of azo chromophores results in a change in $T_{\mathrm{g}}$ of the crosslinked LC polymers. The $T_{\mathrm{g}}$ of the polymer network is higher than room temperature in the trans-form and lower than room temperature in the cis-form. We demonstrate the photoswitchable $T_{\mathrm{g}}$ contribute to the photomechanical bending and a new mechanism for photomechanical bending that attributes the process to an inhomogeneous change in $T_{g}$ of the film is proposed. 
T he development of new materials that can change reversibly in response to external stimuli, such as light, has attracted significant attention from researchers owing to their potential applications in many fields. Light is a clean energy source and a non-contact stimulus, which can be used specifically with regard to wavelength (in the ultraviolet (UV) to infrared (IR) region), intensity, or polarization direction to achieve precise and remote control $^{1-3}$. Light-responsive materials have various applications in photonics, e.g., in optical switching ${ }^{4-6}$, data storage $^{7,8}$, lasing ${ }^{9,10}$, and actuations ${ }^{11-18}$.

Light-responsive materials in which a quick and reversible response can be achieved are of particular interest. However, the development of highly photoresponsive polymers that can convert light into mechanical work in an efficient manner remains a research challenge, which hampers their range of applicability. The difficulty in realizing efficient photomechanical actuations in solid matrices can be attributed to the fact that the performance of functional photochromic molecular switches, such as azobenzene or spirooxazine, is highly sensitive to the viscosity of their polymer matrix. Using fluid environments or lowering the glass transition temperature $\left(T_{\mathrm{g}}\right)$ of the polymer matrix can improve the switching speeds ${ }^{19-21}$ but can compromise other physical properties such as hardness. Thus, a material that can temporarily or reversibly change its $T_{\mathrm{g}}$ under light stimuli is preferable.

For photoresponsive materials, a few azobenzene compounds, that undergo a solid-to-liquid phase transition under photoinduced trans-to-cis isomerization have been reported ${ }^{22-24}$. Most of these compounds required prolonged exposure to strong UV irradiation (e.g., exposure for $30 \mathrm{~min}$ to a light intensity of $\left.40-100 \mathrm{~mW} \mathrm{~cm}^{-2}\right)$ to trigger such a transition ${ }^{23-26}$. Designing new molecules that have high photosensitivity (i.e., the solid-toliquid phase transition occurs within several seconds at a moderate light intensity) remains difficult. Furthermore, the design of such molecular switches with polymerizable groups, such as monomers, is essential for the development of photoactive polymers, rubbers, or gel materials. In contrast to small molecules showing solid-to-liquid phase transitions, polymers exhibit $T_{\mathrm{g}}$ values that determine whether they are glass-like or rubber-like. Polymers with a lower $T_{\mathrm{g}}$ are more flexible and softer at the temperature of their application, and thus respond quickly to mechanical processes such as bending.

To date, little attention has been paid to polymers with photoswitchable $T_{\mathrm{g}}$. Recently, the Wu group found a non-crosslinked azo polymer that shows photoswitchable $T_{\mathrm{g}}$ and reversible solidto-liquid phase transitions ${ }^{27}$. However, photoswitchable $T_{\mathrm{g}}$ in a crosslinked polymer has not been reported. Moreover, the deeper relationship between photomechanical actuations and photoswitchable $T_{\mathrm{g}}$ in azo polymers has never been demonstrated.

In this work, we develop a photoresponsive film based on a LC network using a polymerizable meta-methylazobenzene (M-azo) as the photoresponsive moiety that was covalently crosslinked into a dodecyl glyceryl itaconate (DGI) polymer network (Fig. 1). The free-standing LC film shows a change in its glass transition temperature and reversible photomechanical motions upon exposure to UV/visible light. We found that the $T_{\mathrm{g}}$ of the $\mathrm{LC}$ polymer network decreases after UV absorption; as soon as the UV irradiation is removed, the polymer network recovers its $T_{\mathrm{g}}$ at room temperature upon exposure to visible light or in the dark. The trans-to-cis photoisomerization of azo that crosslinked in the polymer network results in a photoswitchable $T_{\mathrm{g}}$. We demonstrate the reversible change in the $T_{\mathrm{g}}$ of the polymer network contribute to the photomechanical response (e.g. bending) in LC film. Moreover, a mechanism for photomechanical response due to the inhomogeneous $T_{\mathrm{g}}$ change in azo polymer film is proposed.

\section{Results}

Fast photomelting of $\mathrm{M}$-azo monomer at room temperature. The solid-to-liquid phase transition is one of the most fundamental phenomena in material science. It is a process in which the intermolecular forces, such as van der Waals, present in the
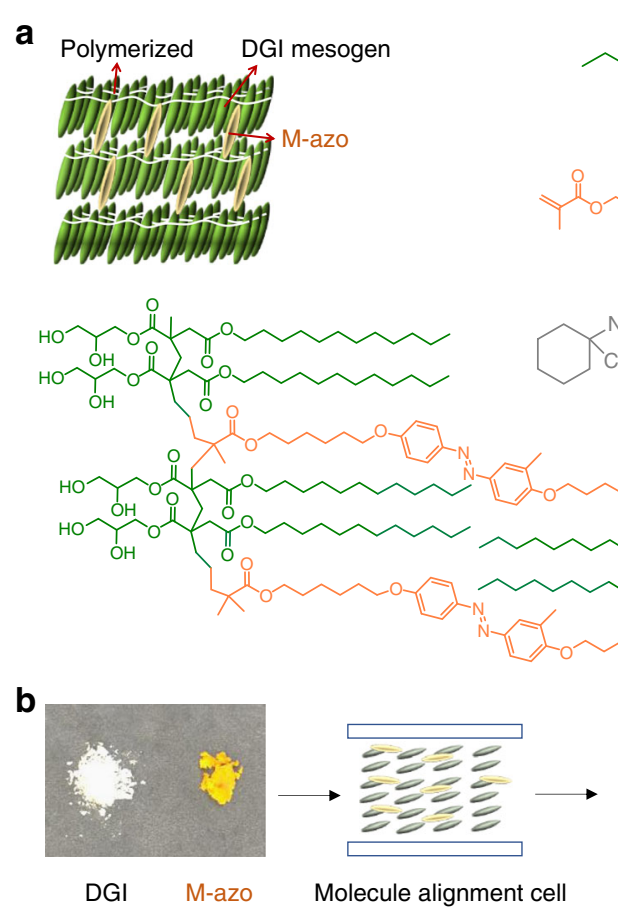

Molecule alignment cell

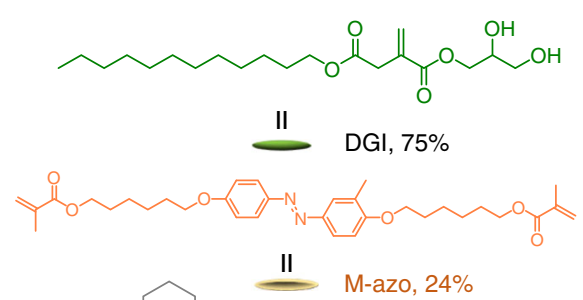

c

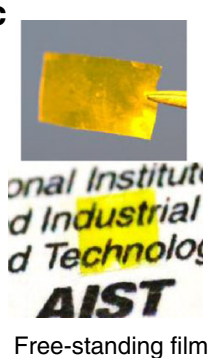

Free-standing film

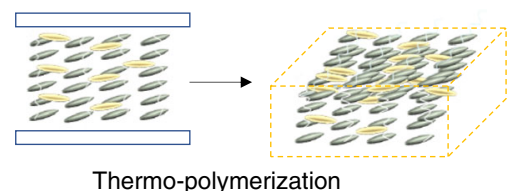

Fig. 1 Schematic representations of molecular structures and their packing modes in the polymer film. a The crosslinked polymer network structure in the film. b Schematic illustration of the fabrication procedure of the polymer film in a molecule alignment cell to realize macroscopic ordering in the film. $\mathbf{c}$ An as-prepared free-standing film that shows high transparency 

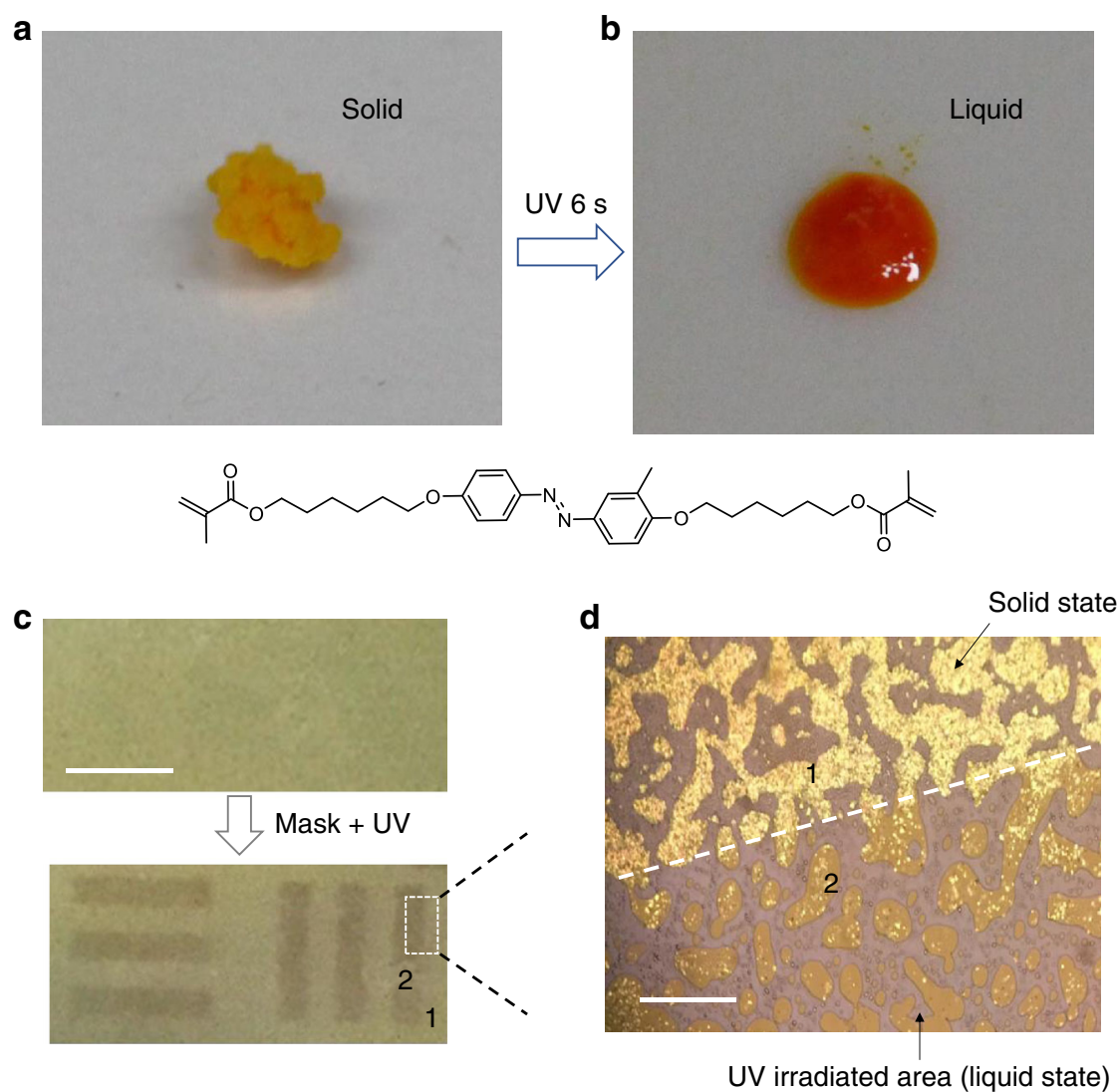

Fig. 2 Photographic images of the M-azo compounds that show photomelting under UV light. $\mathbf{a}, \mathbf{b}$ M-azo compounds before (a) and after (b) UV irradiation ( $\lambda=365 \mathrm{~nm}, 125 \mathrm{~mW} \mathrm{~cm}^{-2}$ ) at room temperature. c A solid-to-liquid phase transition can be observed in the UV-irradiated areas, whereas the regions not irradiated (covered from UV exposure by using a metal mask) retained the solid state. Scale bar, $5 \mathrm{~mm}$. $\mathbf{d}$ Electron microscope image clearly revealing the boundary (dashed-dotted line) between the solid and liquid states in $\mathrm{M}$-azo. Scale bar, $100 \mu \mathrm{m}$

solid state weaken at a certain temperature or pressure. However, little attention has been given to the "photomelting" phenomenon despite a rich functionality of these photomelting compounds $s^{23,28}$. It is therefore exciting to discover that M-azo compounds exhibit rapid photomelting under light irradiation at room temperature. As shown in Fig. 2a, b, the compounds change from a solid to liquid state within $6 \mathrm{~s}$ upon irradiation with UV light $(\lambda=365 \mathrm{~nm})$ at an intensity of $125 \mathrm{~mW} \mathrm{~cm}^{-2}$. By using a metal mask on $\mathrm{M}$-azo under illumination, it was observed that the irradiated regions converted to the liquid phase, while the maskcovered regions retained the solid phase. A clear pattern/ boundary demarcating the two regions can be observed both on the macroscale (Fig. 2c) and microscale (Fig. 2d).

The photomelting process is closely related to molecular packing and weakening of the intermolecular forces during photon absorption ${ }^{29,30}$. As detected under polarized optical microscopy (POM), the M-azo crystals showed bright birefringence patterns before UV irradiation, which indicated an ordered packing of the molecules at room temperature (Supplementary Fig. 1). Upon UV irradiation, the birefringence of the compounds disappeared quickly, which corresponded to the change from crystal phase to isotropic phase (Supplementary Fig. 2). In this process, the basic stereochemical conversion of M-azo molecules is from the extended trans to cis-isomers and involves a change in molecular length from 9 to $5.5 \AA^{31}$. Generally, the photoisomerization process is not accompanied by a solid-to-liquid phase transition. However, for this compound, fast photomelting is observed, which indicates the rapid weakening of intermolecular cohesive forces during photon absorption ${ }^{30}$. This quick phase transition is mainly attributed to meta-substitution in the azo molecular unit, which may significantly change the molecular packing and increase the intermolecular distance (to trigger the solid to liquid change) upon UV irradiation.

To test this, we also synthesized a more common nonsubstituted azo molecule in which the methyl group (of M-azo) was replaced with hydrogen (denoted as $\mathrm{H}$-azo, Supplementary Fig. 3). In contrast with $\mathrm{M}$-azo, $\mathrm{H}$-azo showed no photomelting under identical conditions of light irradiation. As shown in Supplementary Movie 1 and Supplementary Fig. 4, the M-azo and $\mathrm{H}$-azo molecules were placed close to each other with dot sizes of $1-2 \mathrm{~mm}$ on a slide glass and observed by POM at room temperature. Once the UV light $(\lambda=365 \mathrm{~nm}$, intensity $=80 \mathrm{~mW}$ $\mathrm{cm}^{-2}$, illumination area $=2 \times 2 \mathrm{~cm}$ ) was turned on, the M-azo region (right) displayed fast photoinduced melting with disappearing birefringence, whereas the $\mathrm{H}$-azo molecule (left) revealed no change. The photoinduced solid-to-liquid phase transition was not a photothermal process but was due to the photoisomerization, because UV light with an intensity of 80 $\mathrm{mW} \mathrm{cm}{ }^{-2}$ for $10 \mathrm{~s}$ cannot increase the surface temperature to the melting temperature of $\mathrm{M}$-azo $\left(65^{\circ} \mathrm{C}\right)$ or $\mathrm{H}$-azo $\left(73^{\circ} \mathrm{C}\right)$ (Supplementary Fig. 5).

To further evaluate the photosensitivity of the M-azo and $\mathrm{H}$ azo molecules, we used a POM system equipped with an optical spectrophotometer to monitor the kinetics of photochemical phase transitions ${ }^{32}$. As the sample was irradiated with UV light during the experimental measurement, a profile of the kinetic loss in birefringence was simultaneously obtained by the spectrophotometer (Supplementary Fig. 6). The transmittance fade speed is denoted as $T_{1 / 2}$, and is defined as the time taken for the optical density of the specimen to decrease to one-half of its initial value. 
a

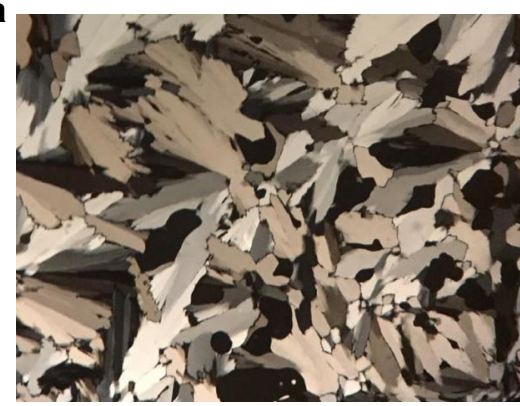

C

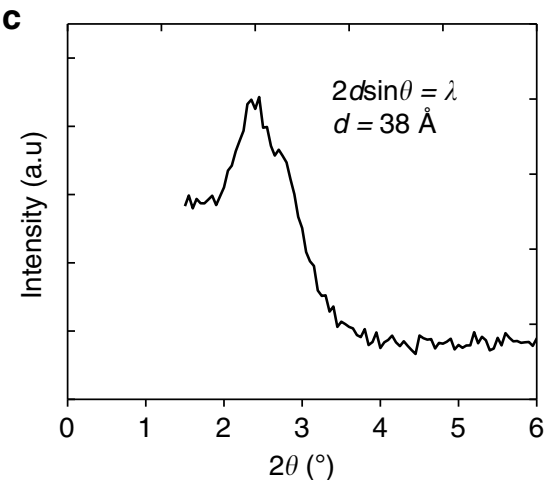

e

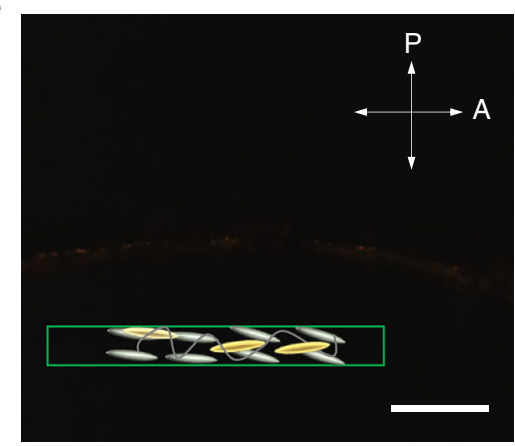

b

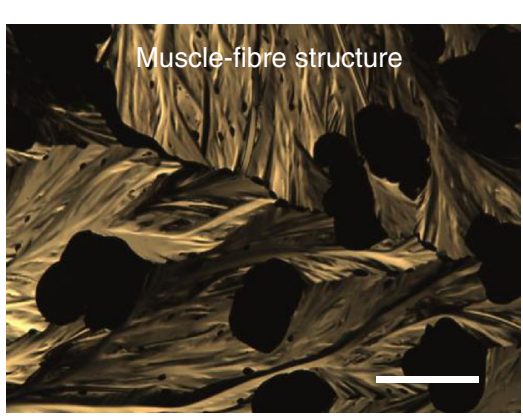

d

DGI bilayer

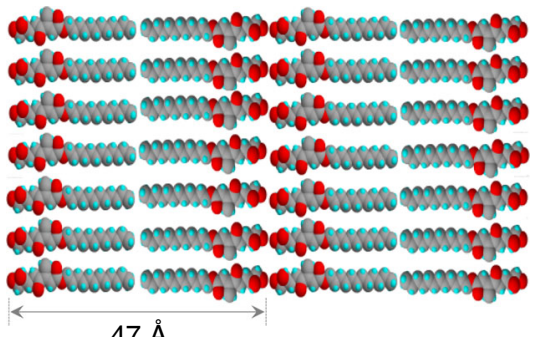

$47 \AA$

f

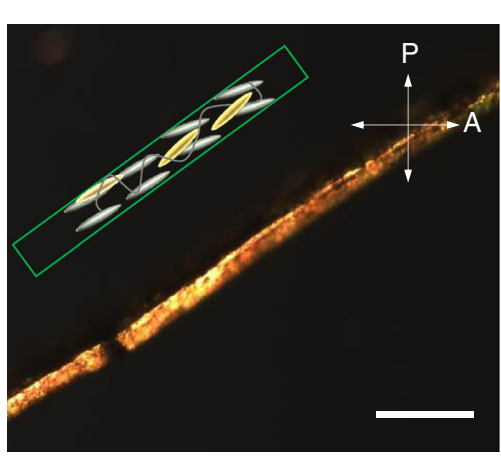

Fig. 3 Structural characterization of DGI monomers and the polymer films. a DGI monomer revealing anisotropic aggregations with a liquid crystal texture under POM. b The patterns in the DGI liquid crystals look similar to those of a muscle-fiber structure under POM. Scale bar, $100 \mu \mathrm{m}$. $\mathbf{c}$ X-ray diffraction pattern of the free-standing polymer film at room temperature that shows a peak at about $2 \theta=2.3^{\circ}$, corresponding to bilayer distances (d) of $\sim 38 \AA$. $\mathbf{d}$ The molecular length of the DGI bilayer shown in the fully extended conformation is about $47 \AA$. The cross-section of the film was tilted at $\mathbf{e} 0^{\circ}$ and $\mathbf{f} 45^{\circ}$ angles relative to the transmission axis of the analyser under POM. The white bidirectional arrows on the top right represent the directions of the analyser ( $\mathrm{A}$ ) and polarizer (P). Scale bars, $30 \mu \mathrm{m}$

In this study, the fade in transmittance speeds was fast for M-azo $\left(T_{1 / 2} \sim 3 \mathrm{~s}\right)$, while $\mathrm{H}$-azo showed no decrease in transmittance during the testing time. The phase transition in M-azo was also confirmed by a 3D laser scanning electron microscope. As shown in Supplementary Fig. 7, the material changes from a solid to liquid state under UV irradiation. To the best of our knowledge, there are no other reports on a polymerizable monomer that can show fast photoinduced solid to liquid phase transition.

Fabrication of free-standing films. We fabricated a free-standing polymer film by free-radical polymerization of $\mathrm{M}$-azo and DGI. DGI was synthesized according to the literature; ${ }^{33}$ it has been previously used in functional hydrogels ${ }^{34-37}$. The DGI molecules displayed smectic LC textures when observed using a POM system (Fig. 3a). Smectic phase is LC phase with a layered structure, which are more ordered than the nematic phase. Three main types of nematic LC monomers have been typically used within the last 10 years to synthesize the photoresponsive polymers that are employed in photomechanical LC films (Supplementary Table 1). This is probably because developing a new polymerizable LC monomer that is applicable to photomechanical polymers is not easy. In this study, the DGI monomer was initially used as a candidate to serve as a muscle-like mesogen moiety that attached as a side chain onto the polymer backbones (Fig. 1a and Fig. 3b). DGI shows no photoresponsive property (Supplementary Fig. 8); the M-azo displays high photosensitivity and serves as a crosslinker. The DGI monomers can self-assemble into lamellar structures due to the presence of the LC phase, and the long alkyl chain (C-12) in DGI and M-azo monomers may enable sufficient free volume to generate a distinct photomechanical response ${ }^{38}$.

During synthesis, the pre-heated precursor mixture containing DGI (22 mg), M-azo (7 mg), and initiator $(0.3 \mathrm{mg})$ was pulled into a molecule alignment cell (area $=2 \times 2 \mathrm{~cm}$, spacing $=5$ or $10 \mu \mathrm{m}$ ) by capillary pressure (Fig. $1 \mathrm{~b}$; for details on synthesis, see Methods). The cell was placed on a hotplate at $60^{\circ} \mathrm{C}$ for $1 \mathrm{~h}$ and then at $125^{\circ} \mathrm{C}$ for $24 \mathrm{~h}$. After cooling to room temperature, the cell was opened with knives and the film was detached from the 
cell with tweezers, yielding a transparent free-standing film with a thickness of 5 or $10 \mu \mathrm{m}$, depending on the spacer thickness (Fig. 1c). The film (denoted as DGI/M-azo) was characterized using X-ray diffraction (XRD) analysis. The peak in the XRD spectrum at $2 \theta=2.3^{\circ}$ indicates that the layer spacing of the lamellar structure was about $3.8 \mathrm{~nm}$, which was smaller than the calculated molecular length of the bilayer $(\sim 4.7 \mathrm{~nm})$ in the fully extended conformation ${ }^{33,34,39}$ (Fig. 3c, d). This indicates that DGI was probably in the smectic C LC phase. To further investigate the molecular orientation along the thickness direction, we also characterized the film using POM. The images revealed a clear contrast for a $45^{\circ}$ angular rotation, whereas they were dark when the angle between the incident light and the sample was $0^{\circ}$ or $90^{\circ}$, which implies that the mesogenic groups had a unidirectional molecular orientation (Fig. 3e, f).

Photoswitchable $\boldsymbol{T}_{\mathbf{g}}$ films. The $T_{\mathrm{g}}$ exists in both amorphous polymers and crystalline polymers. Generally, the $T_{\mathrm{g}}$ of a polymer is related to the temperature at which its molecular chains start to move, and cannot change once the composition and structure of the polymer is fixed. Because of the photoinduced rapid phase transition of $\mathrm{M}$-azo monomers covalently crosslinked into the polymer network, the $T_{\mathrm{g}}$ of the polymer can be reversibly switched under UV/visible light irradiation. Such polymers with photoswitchable $T_{\mathrm{g}}$ were not experimentally demonstrated in literature until very recently ${ }^{27}$. Wu et al. found that a non-crosslinked azo polymer shows a change in $T_{\mathrm{g}}$ from 54 to $-10^{\circ} \mathrm{C}$ after UV irradiation. In this work, DGI/Mazo polymer is a crosslinked polymer network (Supplementary Fig. 9). We measured the $T_{\mathrm{g}} \mathrm{s}$ of the films using differential scanning calorimetry (DSC). As shown in Fig. $4 \mathrm{a}$, the $T_{\mathrm{g}}$ of the DGI/M-azo polymer decreased from 29 (before UV irradiation) to $16^{\circ} \mathrm{C}$ after UV irradiation. The trans-form of the DGI/M-azo films exhibited a higher $T_{\mathrm{g}}\left(29^{\circ} \mathrm{C}\right)$ than room temperature, while the same film in the cis-form shows a lower $T_{\mathrm{g}}\left(16^{\circ} \mathrm{C}\right)$ after UV irradiation. For details on the DSC measurement, the DGI/M-azo films $(\sim 5 \mu \mathrm{m}, 8 \mathrm{mg})$ were cut into small pieces and transferred to the DSC crucible (Supplementary Fig. 10). After measurement, the films were taken out of the crucible and hold for overnight. On the second day, the same pieces of films were irradiated with UV light (intensity $=125 \mathrm{~mW} \mathrm{~cm}^{-2}$ ) on both sides for $2 \mathrm{~min}$ and transferred to a new crucible. The $T_{\mathrm{g}}$ was measured in the same films with cis-form. From the DSC curves, above $T_{\mathrm{g}}$ there is another endothermic peak, which could be attributed to the phase transition of the fundamental components, DGI mesogens (Supplementary Fig. 11). This peak was not detected on the second heating, which was done immediately after first heating and cooling (Supplementary Fig. 12). But this peak appears again after holding the sample overnight, suggesting that the rearrangement of the polymer chains is slow ${ }^{40}$. The change in $T_{\mathrm{g}}$ under UV irradiation was accompanied by an obvious color change of the film from pale yellow to orange, as shown in Fig. 4a (inset).

The degree of change in $T_{\mathrm{g}}$ is determined by the trans to cis ratio in the azo units in the polymer. For a pristine noncrosslinked azo polymer, $T_{\mathrm{g}}$ was decreased by $\sim 60^{\circ} \mathrm{C}$ when more than $90 \%$ of the polymer was in the cis-azo form ${ }^{27}$. In our case, the UV irradiation cannot completely isomerize all the trans Mazo into cis-isomers, especially in the film state. There are two main reasons for this. First, the conversion under continuous UV exposure reaches the photostationary state of azobenzene. The photostationary state of $365 \mathrm{~nm}$ exposure of $\mathrm{M}$-azo (in $\mathrm{CDCl}_{3}$ ) is calculated to be $97 \%$ by nuclear magnetic resonance (Supplementary Fig. 13). Second, in the film state, the strong absorption of trans-azo at $365 \mathrm{~nm}$ allowed the top layer of the film in particular to be converted to cis-azo. The conversion of covalently embedded azobenzene in DGI/M-azo film is $\sim 86 \%$ of trans isomerized into cis after UV irradiation for $35 \mathrm{~s}$ at an intensity of $40 \mathrm{~mW} \mathrm{~cm}^{-2}$ (Supplementary Fig. 14). Thus, the weight content of cis-isomers in the whole film (photostationary state of $365 \mathrm{~nm}$ exposure) is close to $21 \mathrm{wt} \%$ as the total azo content in the film is $24 \mathrm{wt} \%$.

More samples were measured to characterize the $T_{\mathrm{g}}$ change in DGI/M-azo films before and after UV irradiation (Supplementary Fig. 15). Moreover, the $T_{\mathrm{g}}$ value is closely related to the mechanical properties of the polymer films. A higher $T_{\mathrm{g}}$, along with a high storage modulus, results in a high stiffness, which equates to a low percentage elongation when stressed. We then measured the mechanical properties of the DGI/M-azo film before (trans-form) and after UV irradiation (cis-form) by tensile tests (Supplementary Fig. 16). The polymer film before UV irradiation showed a Young's modulus as high as $140 \mathrm{MPa}$. However, the polymer film after UV irradiation (cis-form), showed a much lower Young's modulus, 65.6 MPa but a higher percentage elongation (strain) (Supplementary Fig. 16). This also indicates that the UV light decreased the $T_{\mathrm{g}}$, and thus decreased the Young's modulus of the polymer films.

Photomechanical response in photoswitchable $T_{\mathrm{g}}$ films. Most of the reported photomechanical response times for bulk solid materials (e.g., LC polymers) exposed to strong UV irradiation are on the order of seconds to minutes. For example, the films fabricated using a common $\mathrm{H}$-azo and $\mathrm{LC}$ monomers displayed a slow response, $\sim 30 \mathrm{~s}$ for a UV light intensity of $500-850 \mathrm{~mW} \mathrm{~cm}^{-2}$ with a UV irradiation dose (time $\times$ intensity) of $15-25 \mathrm{~s} \mathrm{~W} \mathrm{~cm}^{-241}$, or several minutes for an intensity of $60-170 \mathrm{~mW} \mathrm{~cm}^{-2}$ (UV irradiation dose $\left.=3.6-10.4 \mathrm{~s} \mathrm{~W} \mathrm{~cm}^{-2}\right)^{13,42}$. Hosono et al. reported on a fast, non-crosslinking polymer actuator that required UV irradiation for $10 \mathrm{~s}$ at an intensity of $300 \mathrm{~W}$ to trigger a photomechanical displacement of $2 \mathrm{~mm}$ with a UV irradiation dose of $3000 \mathrm{~s} \mathrm{~W} \mathrm{~cm}^{-243}$. One of the factors that strongly affects the response time of polymer films is a high $T_{\mathrm{g}}{ }^{21}$. Decreasing the $T_{\mathrm{g}}$ or increasing the softness of the polymer may improve its response time.

As observed above, we found that the $T_{\mathrm{g}}$ value of the DGI/Mazo polymer can be decreased upon UV irradiation and recovered in the dark or by visible light irradiation. The photoresponse of the film was quick which was confirmed by UV/vis spectroscopy. As shown in Fig. 4b, c, the fast decreasing in the absorbance at $365 \mathrm{~nm}$ under UV light and even faster back isomerization process under visible light finished within 2-4 s. The back thermal isomerization is slow in the dark (Supplementary Fig. 17).

The corresponding photoswitchable $T_{\mathrm{g}}$ is beneficial for achieving efficient photomechanical actuations in the polymer network. To further characterize this phenomenon, we inspected the photomechanical response of the DGI/M-azo polymer. As shown in Supplementary Movie 2, the bending and recovery of the polymer film can be repeated by switching on/off the UV and visible light continuously. When the UV light $(\lambda=365 \mathrm{~nm})$ was switched on, the film bent in the direction of the irradiation at the same time, whereas when the blue light $(\lambda=465 \mathrm{~nm})$ was turned on, the film was restored to its flat (original) state immediately. The bending of the film was accompanied by a color change from yellow to orange and then back (orange to yellow). Here, the bending/unbending processes of the film could be controlled and repeated for many cycles without any obvious fatigue (Supplementary Movie 2).

Photoabsorption is a time-dependent process, and the degree of change in $T_{\mathrm{g}}$ and the response speed should be dependent on 

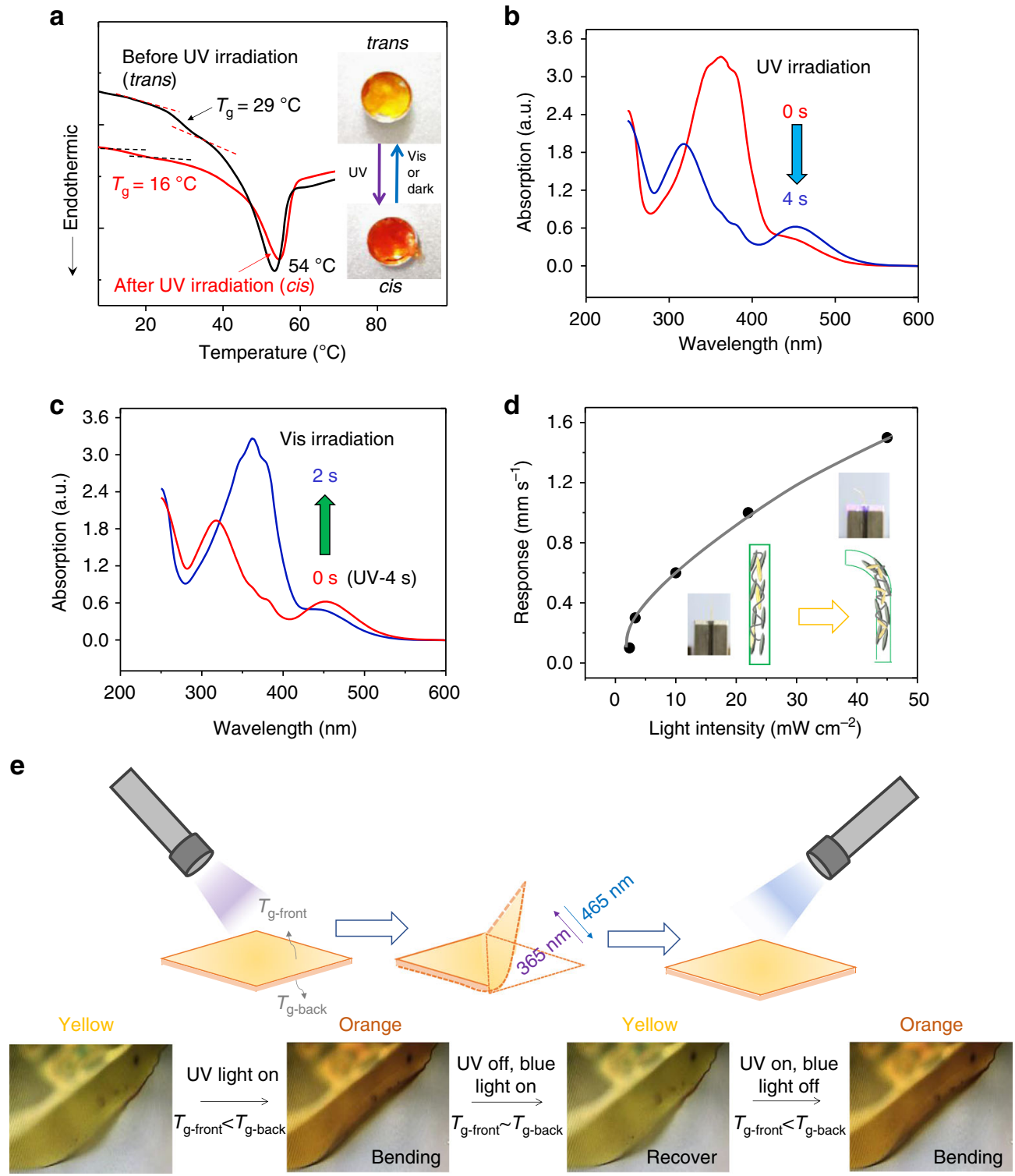

Fig. 4 Photoswitchable $T_{g}$ UV/Vis absorption spectra and photomechanical response of the film. a DSC measurements of $T_{g}$ during the first heating process of the polymer in trans-, and cis-form. $\mathbf{b}$ Absorption spectra of a DGI/M-azo film before (red line) and after (blue line) irradiating with a light of $\lambda=$ $365 \mathrm{~nm}$ for $4 \mathrm{~s}$ (light intensity $=100 \mathrm{~mW} \mathrm{~cm}^{-2}$ ). c Absorption spectra of a DGI/M-azo film before (red line) and after (blue line) irradiation using light of $\lambda$ $=465 \mathrm{~nm}$ for $2 \mathrm{~s}$ (light intensity $=100 \mathrm{~mW} \mathrm{~cm}^{-2}$ ). $\mathbf{d}$ The relationship between the photoresponse speed of the fresh DGI/M-azo fiber and UV light intensity. e The continuous photomechanical response of the polymer film under light irradiation. The UV irradiation $\left(\lambda=365 \mathrm{~nm}, 11 \mathrm{~mW} \mathrm{~cm}^{-2}\right) \mathrm{caused}$ bending of the film; the visible light $\left(\lambda=465 \mathrm{~nm}, 30 \mathrm{~mW} \mathrm{~cm}^{-2}\right)$ subsequently straightened the bent film. $T_{\mathrm{g} \text {-front, }} T_{\mathrm{g}}$ value of the film on the front side; $T_{\mathrm{g}}$ back, $T_{\mathrm{g}}$ value of the film on the backside

the light intensity. To characterize the relationship between the light intensity and the response speed, we applied different intensities of UV light for irradiating a DGI/M-azo polymer fiber. As shown in Fig. 4d and Supplementary Movie 3, the photoresponse speed improved gradually with increasing intensity of the irradiated light. When the light intensity was increased from 3.28 to $45 \mathrm{~mW} \mathrm{~cm}^{-2}$, the bending speed (displacement/ time) improved from 0.4 to $2 \mathrm{~mm} \mathrm{~s}^{-1}$ at a testing temperature of $\sim 20^{\circ} \mathrm{C}$. For DGI/M-azo film, the average irradiation dose is $\sim 0.046 \mathrm{~s} \mathrm{~W} \mathrm{~cm}^{-2}$ for a bending displacement of $3 \mathrm{~mm}$. When the film bent to the direction parallel to the light, it stopped bending and started to oscillate ${ }^{44,45}$. Compared with previously reported photoresponsive films that need a relatively large UV irradiation dose, this polymer can efficiently absorb a small number of photons to cause mechanical reactions.
Mechanism for photomechanical response. XRD analysis and Fourier transform infrared (FT-IR) spectroscopy were employed to study the structural changes occurring inside the polymer during photoabsorption and characterize the film before and after UV irradiation. As shown in Supplementary Fig. 18, there was no obvious change in the peak position and intensity in the XRD pattern or IR spectrum, indicating that the order parameter of the structure had not been significantly altered. In such situations, bending cannot be explained by using the generally reported mechanism alone, which attributes the process to a reduction in the order parameter of the films ${ }^{46}$. Considering these observations, we propose a complementary mechanism for photomechanical bending that attributes the process to an inhomogeneous change in $T_{\mathrm{g}}$ of the film (Fig. 4e). Absorption of photons first occurs on the front surface of the film, and then, the 
residual photons are absorbed in the inner region, which results in a difference in $T_{\mathrm{g}}$ values between the front and back surfaces of the film, i.e., $T_{\mathrm{g} \text {-front }}<T_{\mathrm{g} \text {-back }}$. Thus, a difference in the internal strains, i.e., $\varepsilon_{\text {front }}<\varepsilon_{\text {back }}$, is produced, causing the film to bend in the direction of the incident UV light.

\section{Discussion}

In this work, $\mathrm{M}$-azo compounds showed photomelting within several seconds, exhibiting their ability to quickly overcome the intermolecular forces ${ }^{30}$ during trans-to-cis isomerization. In the trans state, the intermolecular forces are high, such that M-azo exists as a solid; in the cis state, it is liquid due to the intermolecular forces being overcome after UV irradiation. The value of $T_{\mathrm{g}}$ is greatly influenced by the intermolecular forces ${ }^{47}$. Jenekhe et al. reported a direct experimental observation of the effects of intermolecular forces on the $T_{\mathrm{g}}$ of diverse polymers and showed that alleviation of intermolecular forces causes a dramatic shift of the $T_{\mathrm{g}}$ to a lower temperature ${ }^{47}$. Similar to small molecules, the trans and cis configuration azopolymers may have different intermolecular forces, which influence the $T_{\mathrm{g}}$ values.

When the temperature during use, $T_{\text {using }}$, is lower than the $T_{\mathrm{g}}$ of the polymer (i.e., $T_{\mathrm{g}}>T_{\text {using }}$ ), the polymer is in a relatively glassy state. When $T_{\mathrm{g}}$ of the polymer is decreased via specific photon absorption to a value lower than the use temperature (i.e., $T_{\mathrm{g}}<T_{\text {using }}$ ), the polymer is in a rubber state and response speed of the polymer is probably improved.

Moreover, we think the design of highly photoresponsive polymers should first begin from a basic monomer design, because certain properties observed at the molecular level (e.g., fast photomelting during photoisomerization) may be amplified in the polymeric materials with interesting macroscopic properties, such as a high rate of change of $T_{\mathrm{g}}$ by photoswitching. Second, by coupling the highly photoresponsive molecular switches covalently embedded in the ordered parameter of the LC networks (i.e., DGI), the collective molecular motion in ordered materials is amplified ${ }^{11}$.

In summary, we reported the light-induced mechanical response of a LC polymer that show photoswitchable $T_{\mathrm{g}}$. We used an azo crosslinker, $\mathrm{M}$-azo, which shows photoinduced melting, to crosslink the LC monomer DGI. The azo chromophores in the polymer network showed reversible trans-to-cis photoisomerization, resulting in the $T_{\mathrm{g}}$ change of the crosslinked polymer network. The polymer network had a $T_{\mathrm{g}}$ higher than room temperature in the trans-form and lower than room temperature in the cis-form. We further studied the photomechanical actuation of the polymer network and demonstrated that photoswitchable $T_{\mathrm{g}}$ contributes to light-induced bending. Especially, we propose a mechanism for photomechanical response due to an inhomogeneous change in the $T_{\mathrm{g}}$ of the film. We anticipate these results provide a deeper insight in the photomechanical response in azobenzene LC polymers.

\section{Methods}

Materials and analytical methods. The solvents, tetrahydrofuran (THF), $N, N$ dimethylformamide (DMF), toluene, dimethyl sulfoxide-d6 (DMSO-d6), and deuterated chloroform, $\left(\mathrm{CDCl}_{3}\right)$ were purchased from Aldrich and used asreceived. The thermal initiator 1,1'-azobis(cyclohexane-1-carbonitrile) (V-40, chemical structure shown in Fig. 1) was purchased from Wako Pure Chemical Industries Ltd., Japan. Silica gel $(40 \mu \mathrm{m})$ was also purchased from Wako Pure Chemical Industries Ltd., Japan, and used in column chromatography. More details about the materials are shown in the Supplementary Note $1 .{ }^{1} \mathrm{H}$ NMR and ${ }^{13} \mathrm{C}$ NMR spectra were measured using Bruker Advance NMR spectrometers at resonance frequencies of $400 \mathrm{MHz}$ and $500 \mathrm{MHz}$, respectively. Multiplicities are abbreviated as follows: singlet ( $\mathrm{s})$, doublet $(\mathrm{d})$, triplet $(\mathrm{t})$, and multiple $(\mathrm{m})$. Highresolution mass spectrometry (HRMS) was measured using a JEOL spiral TOF JMS-S3000 spectrometer. The light source used was an LED lamp (CCS, HLV24VV365-4W PCLTL for $\lambda=365 \mathrm{~nm}$; HLV2-22 $\beta \mathrm{L}-3 \mathrm{~W}$ for $\lambda=465 \mathrm{~nm}$ ), or a highpower mercury light (REX-250) with different filters. The light intensity was
}

monitored by a Newport 1917-R optical power meter with 818 -ST-UV photodetector or USHIO-accumulated UV meter UIT-250. The surface temperature of the compounds (M-azo) before and after UV irradiation was measured by an infrared thermometer (TG167, FLIR). The 3D laser scanning electron microscopic images were obtained using a Keyence VK-X100 laser microscope with a laser wavelength of $658 \mathrm{~nm}$. DSC thermograms were obtained using SII Nanotechnology DSC6100. More details about the methods are shown in the Supplementary Methods.

Monomer synthesis and characterization. The detailed organic synthesis procedures of $\mathrm{M}$-azo, $\mathrm{H}$-azo, intermediate products, DGI monomer, and their structural characterizations are shown in the Supplementary Note 1.

Film fabrication. LC polymer films were fabricated by copolymerizing M-azo and DGI monomers via thermal initiation. A mixture of the monomers containing 22 $\mathrm{mg}$ DGI, $7 \mathrm{mg} \mathrm{M}$-azo, and $0.3 \mathrm{mg}$ initiator was melted by heating at a temperature of $70^{\circ} \mathrm{C}$ in a vial. A small amount of toluene $(20 \mu \mathrm{l})$ was added to decrease the viscosity of the mixture. The mixture was drawn by capillary pressure into a parallel setup containing molecule alignment cells (thickness $=5 \mu \mathrm{m}$ or $10 \mu \mathrm{m}$, area $=2 \times 2 \mathrm{~cm}, \mathrm{KSRP}-50 /$ A107P1NSS or DONNELLY, E.H.C Co. Ltd), which were pre-heated to $70^{\circ} \mathrm{C}$. For polymerization, the sample was heated at $60^{\circ} \mathrm{C}$ for $1 \mathrm{~h}$ on a hot plate, followed by heating to $125^{\circ} \mathrm{C}$ for $24 \mathrm{~h}$ under a $\mathrm{N}_{2}$ atmosphere to ensure a full conversion of the acrylate monomers.

The entire process was carried out in a lab with $<500 \mathrm{~nm}$ cut-off light. After polymerization, the cell was opened with knives and the DGI/M-azo films were removed from the glass substrates with tweezers.

Film characterization. The molecular alignment of the films was studied by observing their cross-section using an Olympus BX51 POM equipped with a Linkam heating stage. Absorption spectra were recorded on a JASCO V-670 using double-beam spectrophotometer. The XRD spectra were obtained using a SmartLab Rigaku X-ray analytical machine with a $\mathrm{Cu} \mathrm{Ka}(\lambda=1.5418 \AA$ ) source. Tensile stress-strain curves were acquired using a commercial machine (Tensilon EZ-LX, SHIMAZU). FT-IR spectra were recorded on a Perkin-Elmer Spectrum 2000 spectrometer in the wavelength region of $370-4000 \mathrm{~cm}^{-1}$. More detailed descriptions of the characterization methods can be found in the Supplementary Methods.

Data availability. The authors declare that the main data supporting the findings of this study are available within the article and its Supplementary Information files, or available from the corresponding author upon reasonable request.

Received: 29 October 2017 Accepted: 23 July 2018

Published online: 13 August 2018

\section{References}

1. Jiang, B. H., Kelch, S. \& Lendlein, A. Polymers move in response to light. Adv. Mater. 18, 1471-1475 (2006).

2. Priimagi, A., Barrett, C. J. \& Shishido, A. Recent twists in photoactuation and photoalignment control. J. Mater. Chem. C. 2, 7155-7162 (2014).

3. Manrique-juárez, M. D. et al. Switchable molecule-based materials for microand nanoscale actuating applications: achievements and prospects. Coord. Chem. Rev. 308, 395-408 (2016).

4. Huck, N. P. M., Jager, W. F., Lange, B. D. \& Feringa, B. L. Dynamic control and amplification of molecular chirality by circular polarized light. Science 273, 1686-1688 (1996).

5. Hrozhyk, U. A., Serak, S. V., Tabiryan, N. V. \& Bunning, T. J. Optical tuning of the reflection of cholesterics doped with azobenzene liquid crystals. Adv. Funct. Mater. 17, 1735-1742 (2007).

6. Ikeda, T. \& Tsutsumi, O. Optical switching and image storage by means of azobenzene liquid-crystal films. Science 268, 1873-1875 (1995).

7. Toriumi, A., Kawata, S. \& Gu, M. Reflection confocal microscope readout system for three-dimensional photochromic optical data storage. Opt. Lett. 23, 1924-1926 (1998)

8. White, T. J., McConney, M. E. \& Bunning, T. J. Dynamic color in stimuliresponsive cholesteric liquid crystals. J. Mater. Chem. 20, 9832-9847 (2010).

9. Coles, H. \& Morris, S. Liquid-crystal lasers. Nat. Photonics 4, 676-685 (2010)

10. De Sio, L., Tabiryan, N., Bunning, T., Kimball, B. R. \& Umeton, C. Dynamic photonic materials based on liquid crystals. Prog. Opt. 58, 1-64 (2013).

11. Finkelmann, H., Nishikawa, E., Pereira, G. \& Warner, M. A new optomechanical effect in solids. Phys. Rev. Lett. 87, 15501-15504 (2001).

12. Van Oosten, C. L., Bastiaansen, C. W. \& Broer, D. J. Printed artificial cilia from liquid-crystal network actuators modularly driven by light. Nat. Mater. 8, 677-682 (2009). 
13. Iamsaard, S. et al. Conversion of light into macroscopic helical motion. Nat. Chem. 6, 229-235 (2014).

14. Lv, J. et al. Photocontrol of fluid slugs in liquid crystal polymer microactuators. Nature 537, 179-184 (2016)

15. Palagi, S. et al. Structured light enables biomimetic swimming and versatile locomotion of photoresponsive soft microrobots. Nat. Mater. 15, 647-653 (2016).

16. Yu, H. \& Ikeda, T. Photocontrollable liquid-crystalline actuators. Adv. Mater. 23, 2149-2180 (2011).

17. Ikeda, T. Photomodulation of liquid crystal orientations for photonic applications. J. Mater. Chem. 13, 2037-2057 (2003).

18. Bisoyi, H. K. \& Li, Q. Light-driven liquid crystalline materials: from photoinduced phase transitions and property modulations to applications. Chem. Rev. 116, 15089-15166 (2016).

19. Such, G., Evans, R. A., Yee, L. H. \& Davis, T. P. Factors influencing photochromism of spiro-compounds within polymeric matrices. J. Macromol. Sci. 43, 547-579 (2003).

20. Evans, R. A. et al. Photochromic dye switching speeds in a rigid polymer matrix. Nat. Mater. 4, 249-253 (2005).

21. Petr, M. \& Hammond, P. T. Room temperature rapid photoresponsive azobenzene side chain liquid crystal polymer. Macromolecules 44, 8880-8885 (2011).

22. Norikane, Y. \& Yoshida, M. Photoinduced isothermal phase transitions of liquid-crystalline macrocyclic azobenzenes. Chem. Commun. 47, 1770-1772 (2011).

23. Akiyama, H. \& Yoshida, M. Photochemically reversible liquefaction and solidification of single compounds based on a sugar alcohol scaffold with multi azo-arms. Adv. Mater. 24, 2353-2356 (2012).

24. Norikane, Y. et al. Photoinduced crystal-to-liquid phase transitions of azobenzene derivatives and their application in photolithography processes through a solid-liquid patterning. Org. Lett. 16, 5012-5015 (2014).

25. Uchida, E., Azumi, R. \& Norikane, Y. Light-induced crawling of crystals on a glass surface. Nat. Commun. 6, 7310-7316 (2015).

26. Baroncini, M. et al. Photoinduced reversible switching of porosity in molecular crystals based on star-shaped azobenzene tetramers. Nat. Chem. 7, 634-640 (2015).

27. Zhou, H. et al. Photoswitching of glass transition temperatures of azobenzenecontaining polymers induces reversible solid-to-liquid transitions. Nat. Chem. 9, 145-151 (2017).

28. Saito, S. et al. Light-melt adhesive based on dynamic carbon frameworks in a columnar liquid-crystal phase. Nat. Commun. 7, 12094-12100 (2016).

29. Hoshino, M. et al. Crystal melting by light: X-ray crystal structure analysis of an azo crystal showing photoinduced crystal-melt transition. J. Am. Chem. Soc. 136, 9158-9164 (2014).

30. Poborchii, V. V., Kolobov, A. V. \& Tanaka, K. Photomelting of selenium at low temperature. Appl. Phys. Lett. 74, 215-217 (1999).

31. Kumar, G. S. \& Neckers, D. Photochemistry of azobenzene-containing polymers. Chem. Rev. 89, 1915-1925 (1989).

32. Norikane, Y. et al. Photoinduced phase transitions in rod-shaped azobenzene with different alkyl chain length. J. Photopolym. Sci. Technol. 29, 149-157 (2016).

33. Naitoh, K., Ishii, Y. \& Tsujii, K. Iridescent phenomena and polymerization behaviors of amphiphilic monomers in lamellar liquid crystalline phase. J. Phys. Chem. 95, 7915-7918 (1991).

34. Yue, Y., Haque, M. A., Kurokawa, T., Nakajima, T. \& Gong, J. P. Lamellar hydrogels with high toughness and ternary tunable photonic stop-band. Adv. Mater. 25, 3106-3110 (2013).

35. Yue, Y. et al. Mechano-actuated ultrafast full-colour switching in layered photonic hydrogels. Nat. Commun. 5, 4659-4666 (2014).

36. Yue, Y. \& Gong, J. P. Tunable one-dimensional photonic crystals from soft materials. J. Photochem. Photobiol. C. Photochem. Rev. 23, 45-67 (2015).

37. Yue, Y., Li, X., Kurokawa, T., Haque, M. A. \& Gong, J. P. Decoupling dualstimuli responses in patterned lamellar hydrogels as photonic sensors. J. Mater. Chem. B. 4, 4104-4109 (2016).

38. Zhang, Y. et al. Photoinduced bending behavior of crosslinked liquid-crystalline polymer films with a long spacer. J. Mater. Chem. 20, 7123-7130 (2010).
39. Yue, Y. Self-assembled liquid-crystalline membranes form supramolecular hydrogels via hydrogen bonding. Macromol. Rapid Commun. 38, 1600762-1600768 (2017).

40. Tashiro, K., Minagawa, Y., Kobayashi, M., Kawai, T. \& Yoshino, K. Structure and thermochromic solid-state phase transition of poly(3-alkythiophene). J. Polym. Sci. 29, 1223-1233 (1991).

41. Lee, K. M. \& White, T. J. Photomechanical response of composite structures built from azobenzene liquid crystal polymer networks. Polymers 3, 1447-1457 (2011)

42. Iamsaard, S. et al. Fluorinated azobenzenes for shape-persistent liquid crystal polymer networks. Angew. Chem. Int. Ed. 55, 9908-9912 (2016).

43. Hosono, N. et al. Large-area three-dimensional molecular ordering of a polymer brush by one-step processing. Science 330, 808-811 (2010).

44. Gelebart, A. H., Vantomme, G., Meijer, E. W. \& Broer, D. J. Mastering the photothermal effect in liquid crystal network: a general approach for self-sustained mechanical oscillators. Adv. Mater. 29, 1606712-1606717 (2017).

45. Gelebart, A. H., Mulder, D. J., Vantomme, G., Schenning, A. P. H. J. \& Broer D. J. A rewritable, reprogrammable, dual light-responsive polymer actuator. Angew. Chem. Int. Ed. 56, 13436-13439 (2017).

46. Harris, K. D. et al. Large amplitude light-induced motion in high elastic modulus polymer actuators. J. Mater. Chem. 15, 5043-5048 (2005).

47. Jenekhe, S. A. \& Roberts, M. F. Effects of intermolecular forces on the glass transition of polymers. Macromolecules 26, 4981-4983 (1993).

\section{Acknowledgements}

This work was supported by JSPS KAKENHI Grant Numbers JP18K14291, JP16K14069, JP16H04141, JP17H05275 in Scientific Research on Innovative Areas "Photosynergetics".

\section{Author contributions}

Y.Y. conceived the idea, performed the major experiments, and wrote the manuscript. Y. N. designed the experiments on Fig. 4d, Supplementary Fig. 6, and contribute to edit the manuscript. R.A. helped in collecting the data on the FT-IR measurement. E.K. contributed to D.S.C. measurement. All the authors provided suggestions on experiments and improvements.

\section{Additional information}

Supplementary Information accompanies this paper at https://doi.org/10.1038/s41467 018-05744-x.

Competing interests: The authors declare no competing interests.

Reprints and permission information is available online at http://npg.nature.com/ reprintsandpermissions/

Publisher's note: Springer Nature remains neutral with regard to jurisdictional claims in published maps and institutional affiliations.

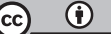

Open Access This article is licensed under a Creative Commons Attribution 4.0 International License, which permits use, sharing, adaptation, distribution and reproduction in any medium or format, as long as you give appropriate credit to the original author(s) and the source, provide a link to the Creative Commons license, and indicate if changes were made. The images or other third party material in this article are included in the article's Creative Commons license, unless indicated otherwise in a credit line to the material. If material is not included in the article's Creative Commons license and your intended use is not permitted by statutory regulation or exceeds the permitted use, you will need to obtain permission directly from the copyright holder. To view a copy of this license, visit http://creativecommons.org/ licenses/by/4.0/.

(C) The Author(s) 2018 